\title{
Desarrollo Humano en las provincias argentinas 2004-2008. Una mirada desde el enfoque territorial
}

\author{
María Albina Pol \\ Universidad Nacional de Cuyo - CONICET, Mendoza, Argentina. \\ Email: albinapol@yahoo.com.ar
}

Resumen: Las libertades de que disponen las personas se encuentran condicionadas por el entorno en el que cotidianamente realizan sus actividades. El territorio se constituye entonces en determinante del espacio de oportunidades entre las que cada individuo puede elegir. Esto lleva a enfatizar la importancia de examinar las capacidades humanas en entidades geográficas específicas.

En el artículo se retoma el Índice de Desarrollo Humano Territorial (IDHT), medida elaborada con el propósito de aumentar la validez del IDHPNUD para dar cuenta de las brechas espaciales a escala subnacional, y se aplican al mismo algunas modificaciones a fin de lograr una aproximación actualizada y más rigurosa al panorama del desarrollo humano en las provincias argentinas.

Se detallan en primer lugar los ajustes metodológicos realizados al IDHT y se presentan luego los resultados de su aplicación a las provincias argentinas, describiendo la evolución que ha experimentado el desarrollo humano en dos puntos en el tiempo (2004-2008).

Palabras clave: desarrollo humano, territorio, provincias argentinas.

\section{Human Development in argentine provinces 2004-2008. A view from the territorial approach}

Abstract: The freedoms that people have are conditioned by the environment in which they operate daily. Thus, the territory constitutes itself in the determinant of the space of opportunities from which each individual can choose. This leads to emphasize the importance of examining human capabilities within specific geographic entities.

The article goes back to the Territorial Human Development Index (TDHI), measure elaborated in order to increase the validity of the HDI-UNDP to give account of the spatial gaps, at subnational scale, and some modifications are applied in order to have an updated and more rigorous approximation to the human development overview in the argentine provinces.

First, the paper describes the methodological adjustments made to THDI. Then, the results of its application to the Argentine provinces are presented, describing the evolution experienced by human development at two points in time (2004-2008).

Keys words: human development, territory, argentine provinces. 


\section{Desenvolvimento Humano nas províncias argentinas 2004-2008. Uma visão a partir da abordagem territorial}

Resumo: As liberdades disponíveis aos indivíduos são condicionados pelo ambiente em que operam diariamente. O território é, então, determinar a área de oportunidades a partir do qual cada indivíduo pode escolher. Isto leva a enfatizar a importância de considerar as capacidades humanas em determinadas entidades geográficas. O artigo toma Territorial Índice de Desenvolvimento Humano (IDHT), elaboradas a fim de aumentar a validade do IDH-PNUD para explicar lacunas do território a nível subnacional, e aplicar as mesmas modificações para alcançar uma abordagem atualizada e mais rigorosa para o panorama do desenvolvimento humano nas províncias. Primeiro detalhados feitos ajustes metodológicos para IDHT e depois apresentar os resultados de sua aplicação para as províncias do país, descrevendo a evolução do desenvolvimento humano passou por dois pontos no tempo (2004-2008).

Palavras-chave: Desenvolvimento humano, território, províncias argentinas.

$* * *$

\section{Introducción}

En décadas recientes la noción de desarrollo como proceso multidimensional orientado a expandir el bienestar de las personas ha ido adquiriendo fuerza. Esto se ha debido no sólo a la propia consistencia de los argumentos, sino también a la notable insuficiencia de las teorías tradicionales para explicar la persistencia y profundización de las desigualdades.

Entre las nuevas perspectivas, el enfoque del desarrollo humano pone el énfasis en la ampliación de las libertades para que los individuos lleven adelante la vida que desean y valoran. Estas libertades se encuentran condicionadas por el entorno en el que las personas se desenvuelven cotidianamente, según el lugar donde vivan y lleven a cabo sus actividades, será el espectro y tipo de opciones que tendrán a su alcance.

El territorio es entonces un agente determinante en la configuración del espacio de oportunidades al que las personas pueden acceder, lo que lleva a plantear la importancia de analizar el desarrollo humano en unidades espaciales específicas. Se requiere evaluar el nivel de logros existente en entornos particulares y, a partir de ello, obtener la amplitud de las brechas a recorrer para equiparar las oportunidades de las personas. Este tipo de información resulta una herramienta fundamental en la identificación de áreas y territorios prioritarios para el diseño de políticas que respondan a las demandas específicas de los diferentes grupos poblacionales.

Sobre la base de esa argumentación, en estudios anteriores ${ }^{1}$ realizamos un análisis que nos permitió constatar la escasa capacidad del Índice de Desarrollo Humano (IDH) elaborado por el Programa de las Naciones Unidas para el Desarrollo (PNUD) para reflejar las disparidades al interior 
de países con un relativamente alto nivel de logros. Ello nos llevó a proponer una medida con mayor validez discriminativa en términos espaciales, el Índice de Desarrollo Humano Territorial (IDHT). La aplicación del IDHT a las provincias argentinas y los estados mexicanos mostró, de manera más aproximada, el panorama de la desigualdad en esas naciones y arrojó elementos que reafirman la importancia de la dimensión territorial en la configuración de las oportunidades.

Es propósito de este trabajo realizar una revisión del IDHT efectuando algunos ajustes orientados a aumentar su validez para captar, de manera específica, el patrón de distribución de las capacidades humanas entre las provincias argentinas e introduciendo como dimensión de análisis el cambio en el tiempo. La intención es no sólo concentrarse en los mayores o menores niveles de desarrollo y desigualdad al interior del país, sino agregar el análisis de los distintos niveles de avance para cada jurisdicción provincial. Esto teniendo en cuenta que Argentina se ubica, bajo la definición del PNUD, en el grupo de naciones de alto desarrollo humano y en el primer lugar entre los países de la región. El análisis que se presenta muestra que esta visión agregada esconde importantes desigualdades que se amplían a medida que se desciende en la escala espacial.

Se detallan, en primera instancia, los ajustes metodológicos realizados al IDHT y se presentan luego los resultados de su aplicación a las provincias argentinas, describiendo la evolución que ha experimentado el desarrollo humano en dos puntos en el tiempo. Para examinar los avances y retrocesos ocurridos durante el período 2004-2008, se analizan los cambios más notables en los niveles de desarrollo en las jurisdicciones provinciales.

\section{La naturaleza territorial del desarrollo humano}

El desarrollo humano es expandir las libertades de las personas. Es generar cada vez más opciones de vida entre las cuales elegir. Esta perspectiva se distancia de los enfoques tradicionales en el sentido de colocar el énfasis en la eliminación de los obstáculos que dificultan o impiden las posibilidades de ser o actuar de acuerdo con los propios objetivos y no en la mera acumulación de recursos materiales. Las demandas de los individuos no son juzgadas en función de los bienes que poseen, sino de la libertad con la que pueden realmente elegir la vida que están en condiciones de valorar. Es esa libertad la que Sen $(2000,122)$ llama capacidad del individuo de realizar diversas combinaciones de funcionamientos, lo que se valora son las posibilidades a las que efectivamente pueden acceder los individuos en un determinado tiempo y lugar.

Esa relatividad histórico-espacial del desarrollo humano implica que las capacidades de decisión y elección están condicionadas por el entorno inmediato. El modo en que los individuos logran insertarse en su medio y el tipo de vínculos que allí establecen condicionan de manera crucial sus planes de vida y las alternativas para alcanzarlos (PNUD-México 2004). En 
otras palabras, el lugar donde viven, las relaciones que establecen y los recursos disponibles en el medio, son factores determinantes del espectro y tipo de opciones que las personas tienen a su alcance. Por tanto, las oportunidades, su valoración y selección, difieren según las circunstancias que las rodean.

En este punto, la concepción del desarrollo como expansión de las libertades se enlaza con la noción del desarrollo como proceso territorialmente localizado, en el que el entorno espacial es concebido como una construcción dinámica resultado de las múltiples, complejas y particulares relaciones que se establecen entre factores de diversa índole (Rofman 2006).

La especificidad del territorio en que viven lleva a las personas a establecer determinado tipo de vínculos entre sí y con las instituciones más próximas, a ejercer sus libertades y realizar proyectos que a su vez modifican ese entorno inmediato (Barreiro Cavestany 2007). El entramado territorial es el ámbito en el que las libertades se expanden o limitan, es el espacio donde el proceso de desarrollo humano, con su centralidad en el bienestar de las personas y su agencia en los individuos y comunidades, tiene lugar.

Estas consideraciones llevan a sugerir que el conocimiento de la distribución espacial de las capacidades humanas, constituye un primer paso esencial para la identificación de territorios y áreas prioritarios hacia los cuales dirigir estrategias que tiendan a igualar las oportunidades de las personas. Desafío que adquiere especial relevancia en los países de América Latina donde la desigualdad, en sus diferentes dimensiones, aparece como una característica estructural.

La naturaleza local del desarrollo humano exige entonces examinarlo en entidades geográficas específicas. Este requisito se enfrenta con la imposibilidad de definir "lo local” de forma unívoca, ya que la idea puede asociarse con diferentes criterios: ubicación de las concentraciones demográficas, identidades culturales, redes sociales, aglomeraciones económicas o unidades geopolíticas. Dado que las divisiones administrativas al interior de los países constituyen las unidades de gobierno a través de las que se canalizan las diferentes políticas, y que la construcción de información estadística considera como referencia a esas entidades, se ha escogido ese criterio para la elaboración y seguimiento de los indicadores de desarrollo humano.

\section{Metodología para la medición del desarrollo humano a escala territorial}

Numerosos son los debates conceptuales y ejercicios empíricos orientados a avanzar en la operacionalización de las capacidades consideradas fundamentales para el desarrollo de las personas. Uno de los intentos más 
aceptados por los medios políticos y técnicos ha sido el IDH, medida propuesta en su primer informe mundial por el PNUD con la intención de evaluar un conjunto de capacidades básicas en un vasto abanico de países.

El IDH aproxima el desarrollo humano tomando en consideración tres dimensiones la longevidad, el conocimiento y el acceso a recursos. Calcula el logro en cada una de ellas respecto a valores de referencia y luego promedia los indicadores de cada dimensión, obteniéndose un índice con valores que oscilan entre cero y uno. Para la medición a escala de países el IDH-PNUD contempla la esperanza de vida al nacer, la tasa de alfabetización de adultos, la tasa de matriculación combinada primaria, secundaria y superior y el PIB per cápita.

En trabajos anteriores ${ }^{2}$ nos abocamos a argumentar, dado el objetivo que lo orienta y, por tanto, la estructura metodológica en que se sostiene, la escasa sensibilidad del IDH-PNUD para dar cuenta de las disparidades del desarrollo humano a escala subnacional, especialmente en países que han alcanzado un nivel relativamente alto de desarrollo. El análisis arrojó como resultado que algunos de los indicadores, caso de la esperanza de vida al nacer y la tasa de alfabetización de adultos, se tornan territorialmente homogéneos a medida que los países adquieren mayores logros, perdiendo así capacidad para reflejar las brechas que separan a las entidades subnacionales.

Esa constatación nos llevó a proponer una nueva medida con mayor capacidad discriminativa en términos espaciales. El Índice de Desarrollo Humano Territorial (IDHT) contemplaba las mismas tres dimensiones y se ajustaba a la estructura metodológica utilizada para el cálculo del IDHPNUD, pero incorporaba algunas modificaciones basadas en criterios de validez, disponibilidad de información y sensibilidad territorial en lo que refiere a los indicadores componentes.

En la dimensión salud, la esperanza de vida al nacer fue reemplazada por la tasa de supervivencia en menores de cinco años. En el componente educativo, la tasa de alfabetización de adultos fue sustituida por el nivel educativo de personas de 25 años y más y la tasa de matriculación combinada restringida a los niveles de primaria y secundaria. Finalmente, para medir la dimensión nivel de vida, se generó un índice compuesto que, en lugar del PIB per cápita, utilizaba el ingreso per cápita familiar y un indicador de dificultades laborales ${ }^{3}$.

La aplicación de ese nuevo índice a las entidades subnacionales de México y Argentina permitió corroborar la mayor sensibilidad de la medida para aproximar la forma en que se distribuyen las capacidades humanas básicas al interior de dos territorios nacionales que se ubican entre los de mayor desarrollo humano de la región. El IDHT demostró mayor validez para aproximar las brechas que distancian a los estados mexicanos y a las provincias argentinas tanto en el índice agregado como en cada una de sus dimensiones componentes. 
En este artículo se realiza una revisión del IDHT y se proponen, a partir de las conclusiones ya obtenidas, algunos ajustes a la composición y cálculo del mismo, a fin de incrementar su validez para brindar un panorama actualizado de las capacidades humanas en las provincias argentinas. El supuesto que sostiene estas indagaciones es que la situación relativamente ventajosa en la que se ubica Argentina, estaría ocultando la desigual distribución de las oportunidades entre las entidades que la componen y que, si bien con algunas limitaciones, existe información que permite construir indicadores válidos y confiables para medir y analizar dichas disparidades.

\section{Ajustes metodológicos al IDHT}

Las modificaciones al IDHT implican una pérdida en la posibilidad de efectuar comparaciones con otros países o unidades geográficas, sin embargo, dan mayor validez a la medida para aportar pistas sobre la realidad nacional del desarrollo humano, sirviendo en la priorización y despliegue de acciones ajustadas a los desafíos que Argentina está en condiciones de imponerse.

Ante la inexistencia de fuentes que provean datos actualizados y homogéneos a escala provincial, se decidió seleccionar y elaborar los indicadores utilizando la Encuesta Permanente de Hogares (EPH). La EPH es una herramienta que recaba datos sobre los hogares y las personas cubriendo un total de 31 aglomerados urbanos de todo el país. Por tanto, las variables contempladas en el ajuste al IDHT han sido trabajadas de manera tal que representan las principales zonas urbanas de cada una de las provincias argentinas ${ }^{4}$. Esto a excepción del indicador de salud para el que, por la naturaleza de la variable seleccionada, se han considerado los totales provinciales.

Aún contemplando los sesgos producto de esas restricciones, los resultados que se obtuvieron alcanzan una alta significatividad para brindar un panorama del desarrollo humano y sus componentes al interior del territorio argentino. Considerar el área de cobertura de la EPH implica abarcar aproximadamente un 65\% del total de la población del país.

La información provista por la encuesta se utilizó para realizar comparaciones entre provincias en dos momentos en el tiempo, 2004 y 2008, la misma refiere a las poblaciones urbanas de veintidós jurisdicciones más la capital nacional, la provincia de Chubut fue excluida del análisis ya que en 2004 la EPH no contemplaba aglomerados urbanos ubicados en esa entidad administrativa ${ }^{5}$.

Se describen a continuación las modificaciones y criterios metodológicos adoptados en la construcción de cada una de las dimensiones componentes. 


\section{Dimensión salud}

Para elaborar la dimensión sanitaria, se utilizó la tasa de supervivencia en menores de cinco años (1-tasa de mortalidad en menores de cinco años). La fuente de la que se extrajeron los datos fueron los Anuarios Estadísticos elaborados por el Ministerio de Salud de la Presidencia de la Nación.

Los valores máximo y mínimo considerados para la estandarización de la variable suponen una alteración respecto de los utilizados en el IDHT original; por cuanto se procuró aumentar la capacidad del indicador para mostrar las disparidades sanitarias entre las provincias argentinas. El valor mínimo para el cálculo es 3 por mil, a partir de las tasas observadas en los países más desarrollados y el máximo es de 70 por mil considerando las tasas de mortalidad más altas registradas en los países de América Latina ${ }^{6}$. Se entiende que estos valores permiten realizar estimaciones que aproximan la situación sanitaria en la que se encuentran las provincias argentinas en el contexto regional e indican la brecha que aún tienen por recorrer en relación con el comportamiento registrado por los países más avanzados del mundo.

\section{Dimensión educación}

A través del componente educativo se busca medir dos aspectos, la capacidad del propio sistema para satisfacer las demandas educativas de las personas (cobertura) y el capital educativo acumulado en la población bajo estudio (impacto). Para dar cuenta del primero de esos elementos se utilizó, en sustitución de la tasa de matriculación, un proxy de la tasa neta de asistencia escolar para los niveles de primaria y secundaria, calculado en función de los datos de la EPH. Esta decisión fue adoptada a partir de evaluar las estadísticas educativas disponibles y obtener como resultado su alta heterogeneidad y escasa validez para reflejar la situación de cada provincia. Después de analizar la coherencia de los datos obtenidos por contraste con otras fuentes se especificó la variable a considerar. Los valores máximo y mínimo fueron $100 \%$ y $0 \%$ respectivamente, por entender que la meta debe apuntar a la cobertura total en lo que refiere a la asistencia escolar de niños y jóvenes de 6 a 17 años de edad.

Como indicador de impacto se resolvió mantener el de nivel educativo pero medido a partir del porcentaje de personas de 25 a 64 años con secundario completo o más. La variación respecto de la propuesta original aparece en la delimitación del rango, que esta vez coloca la cota superior en 64 años haciéndolo corresponder con la población en edad activa. El valor máximo utilizado fue $85 \%$ de acuerdo con las cifras registradas en los países más desarrollados ${ }^{7}$ y el valor mínimo $0 \%$.

Las ponderaciones de las variables para el cálculo del subíndice fueron de 1/3 para la tasa de asistencia escolar y 2/3 para el nivel educativo, 
mismos pesos otorgados a la tasa de alfabetismo y la tasa de matriculación combinada respectivamente en el IDH-PNUD ${ }^{8}$.

\section{Dimensión nivel de vida}

Para el cálculo de la tercera dimensión se consideró el ingreso per cápita mensual de los hogares ajustado por PPA en dólares. Aplicando los mismos criterios considerados en la construcción del IDH-PNUD, se normalizó la variable utilizando los logaritmos con valores máximo y mínimo de referencia US\$40.000 y US\$100 ${ }^{9}$.

El indicador de ingreso aproxima las posibilidades efectivas de acceso a bienes materiales pero no brinda información respecto de la forma en que esas oportunidades se distribuyen entre la población. Con el propósito de ajustar los valores y reflejar ese último aspecto se incorporó al cálculo de la dimensión una medida de dificultades laborales construida a partir del porcentaje de la PEA que no se encuentra desocupada ni en situación de subocupación horaria. Los valores máximo y mínimo fueron 100\% y 0\% respectivamente. El índice sintético se obtuvo calculando el promedio simple de las dos variables consideradas; ingreso per cápita e indicador laboral.

Las fórmulas de cálculo son las mismas que se aplican para la construcción del IDH-PNUD. Se normaliza el desempeño en cada componente para que asuma valores entre cero y uno, se agregan los indicadores de cada dimensión considerando las respectivas ponderaciones y se calcula el IDHT a partir del promedio simple de los tres subíndices.

\section{Panorama del desarrollo humano en las provincias argentinas}

Aunque los cambios aplicados a las variables impiden la estricta comparación de los resultados de este trabajo con los obtenidos por el PNUD, los datos que indican la situación del país en el concierto mundial brindan un marco pertinente para contextualizar inicialmente el análisis.

Según el último informe mundial del PNUD, Argentina alcanzó en 2005 un IDH de 0,869, cifra que la coloca dentro del grupo de naciones con alto desarrollo humano (IDH igual o superior a 0,800) ubicándose en el lugar 38 de un total de 177 países y en la primera posición entre las naciones de América Latina. En cuanto a las dimensiones componentes, el índice de educación registraba el valor más elevado, 0,947, seguido por el componente sanitario, 0,831 y finalmente la medida de nivel de vida, 0,828. El análisis de la evolución temporal indica que Argentina ha experimentado un continuado proceso de mejora en sus logros, su IDH ha aumentado 1,10 veces en el transcurso de los últimos 30 años (en 1975 tenía un IDH de 0,790). 
La mayor exigencia de los indicadores considerados en el cálculo del IDHT hace que el país asuma en 2008 un valor para ese índice inferior al obtenido según la metodología del PNUD, 0,793 . Aunque muy próxima a la cota superior del rango, esa cifra ubica a Argentina en el grupo de desarrollo humano medio (entre 0,500 y 0,799 ) reflejando el camino que aún tiene por recorrer en la expansión de las oportunidades al alcance de su población. A diferencia de lo registrado por el IDH-PNUD, según el IDHT el mayor nivel de logros se registra en el aspecto sanitario con un valor de 0,828 , seguido por el de nivel de vida 0,823 , y por último el de educación 0,729 . En otras palabras, si las oportunidades educativas se miden de acuerdo con parámetros más ajustados a la realidad del desarrollo del país, éstas aparecen como el aspecto más deficitario.

Al ampliar el panorama y analizar la situación del desarrollo humano en las provincias argentinas, se encuentran las desigualdades ocultas tras esos valores agregados. Las cifras obtenidas en este estudio muestran que, en el año 2008, si bien ninguna provincia se ubica en el nivel de bajo desarrollo (menos de 0,500), la distancia entre las entidades con mayor y menor nivel de logros es aún considerable: Ciudad de Buenos Aires, con un IDHT de 0,905, tiene 1,27 veces el nivel de desarrollo de Formosa, que registra un valor similar al IDH que en 2005 alcanzaban países como Indonesia o Siria, ubicados en los puestos 107 y 108 de la clasificación.

Gráfico 1. Valores extremos IDHT y componentes. 2008.

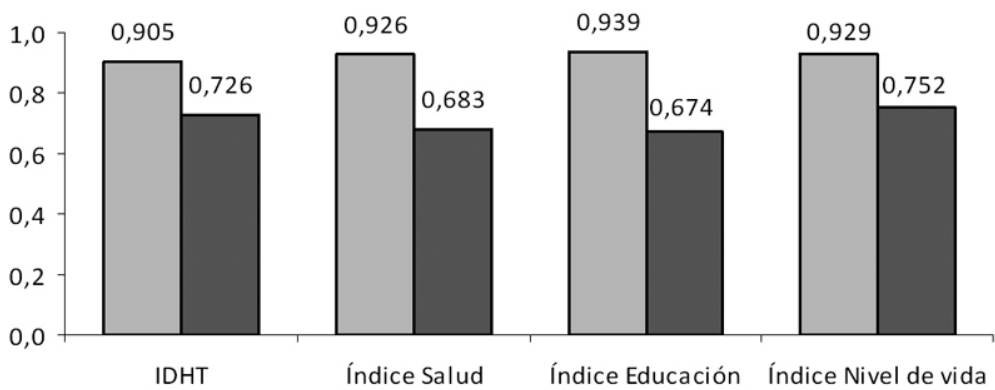

Las distancias varían al considerar los componentes del IDHT, la brecha educativa resulta la más significativa, en esa dimensión los logros de Ciudad de Buenos Aires son 1,39 veces mayores que los de Misiones, sigue el componente sanitario en el que Tierra del Fuego se distancia 1,36 veces de Formosa, mientras que en la dimensión nivel de vida Santa Cruz obtiene un índice 1,24 veces mayor que el de Salta. Estas cifras muestran que, a nivel nacional, la educación aparece no sólo como el componente más deficitario sino también como el más desigualmente distribuido.

El examen de la situación de cada provincia en la clasificación del IDHT y su comparación con la ubicación alcanzada en los componentes 
permite identificar el área en que las entidades registran mayor o menor rendimiento relativo. En relación con las cinco provincias mejor posicionadas según su IDHT, Ciudad de Buenos Aires ocupa el primer lugar, lo que responde principalmente al alto valor alcanzado en el componente educativo. El buen comportamiento de Tierra del Fuego en salud y nivel de vida la ubica en la segunda posición del ranking nacional. Santa Cruz alcanza el mayor valor en el último componente, principal factor explicativo del tercer lugar que ocupa en la clasificación general. Neuquén, que se ubica en el cuarto puesto, posee un rendimiento medio en lo referente a los aspectos educativos. Mendoza, quinta provincia en el ordenamiento, alcanza valores más altos en los logros sanitarios y educativos que en los referentes a nivel de vida.

\section{Cuadro 1. IDHT e índices componentes, posición en el ranking provincial 2008}

\begin{tabular}{|l|c|c|c|c|}
\hline Provincia & IDHT & $\begin{array}{c}\text { Indice } \\
\text { Salud }\end{array}$ & $\begin{array}{c}\text { Indice } \\
\text { Educación }\end{array}$ & $\begin{array}{c}\text { Indice } \\
\text { Nivel de Vida }\end{array}$ \\
\hline Ciudad de Bs As & 1 & 3 & 1 & 3 \\
\hline Tierra del Fuego & 2 & 1 & 5 & 2 \\
\hline Santa Cruz & 3 & 6 & 6 & 1 \\
\hline Neuquén & 4 & 2 & 11 & 4 \\
\hline Mendoza & 5 & 4 & 4 & 8 \\
\hline Córdoba & 6 & 9 & 8 & 11 \\
\hline Río Negro & 7 & 8 & 20 & 5 \\
\hline Santa Fe & 8 & 7 & 13 & 17 \\
\hline La Pampa & 9 & 15 & 12 & 6 \\
\hline San Luis & 10 & 11 & 18 & 7 \\
\hline Buenos Aires & 11 & 10 & 14 & 13 \\
\hline Stgo. del Estero & 12 & 5 & 16 & 19 \\
\hline Catamarca & 13 & 19 & 7 & 14 \\
\hline Entre Ríos & 14 & 12 & 19 & 10 \\
\hline San Juan & 15 & 14 & 9 & 18 \\
\hline La Rioja & 16 & 18 & 10 & 15 \\
\hline Salta & 17 & 20 & 2 & 23 \\
\hline Corrientes & 18 & 21 & 3 & 20 \\
\hline Tucumán & 19 & 13 & 17 & 21 \\
\hline Misiones & 20 & 16 & 23 & 12 \\
\hline Jujuy & 21 & 17 & 15 & 22 \\
\hline Chaco & 22 & 22 & 22 & 16 \\
\hline Formosa & 23 & 23 & 21 & 9 \\
\hline & & & & \\
\hline
\end{tabular}

Si se centra el análisis en las cinco provincias menos favorecidas, se encuentra que Formosa, a pesar de ser la jurisdicción con menor nivel de logros en el IDHT, alcanza una posición ventajosa en el componente nivel de vida, lo que se explica por el alto valor alcanzado en el indicador laboral. Chaco, posicionada en el penúltimo lugar de la tabla, registra un mejor rendimiento en el tercer componente que en las otras dimensiones, lo contrario sucede con Jujuy, ubicada en la posición 21, que posee el segundo más bajo índice de nivel de vida. El puesto 20, ocupado por Misiones, responde principalmente a sus escasos logros educativos. El aspecto que incide en mayor medida en el puesto 19 en el que se ubica Tucumán es el componente de nivel de vida. 
Esta breve descripción, de la que se podrían extraer muchas otras observaciones, permite apreciar que las capacidades básicas se realizan y combinan de forma diferente en los distintos territorios y que, si bien existe cierta correspondencia en los logros alcanzados en cada dimensión ninguna de ellas puede, de manera aislada, explicar el nivel de desarrollo alcanzado por cada jurisdicción.

\section{Los cambios en el desarrollo humano entre 2004 y 2008}

Al analizar comparativamente las series del IDHT para 2004 y 2008, se observa que en ambas clasificaciones se mantiene la regionalización puesta en evidencia por diferentes estudios orientados a examinar el perfil de desarrollo espacial de la República Argentina ${ }^{10}$. Esas investigaciones indican que las provincias norteñas constituyen la región más desfavorecida; mientras que las jurisdicciones del sur nacional y Ciudad de Buenos Aires, presentan los indicadores más favorables.

En correspondencia con esas observaciones, Ciudad de Buenos Aires, Tierra del Fuego, Santa Cruz y Neuquén, se colocan en la cima de las dos clasificaciones generadas a partir del IDHT. Misiones, Tucumán, Jujuy, Formosa y Chaco, aunque con leves variaciones en los lugares ocupados en uno y otro año, aparecen como las circunscripciones con menor desarrollo humano. Entre esos dos extremos se ubica el resto de las provincias argentinas.
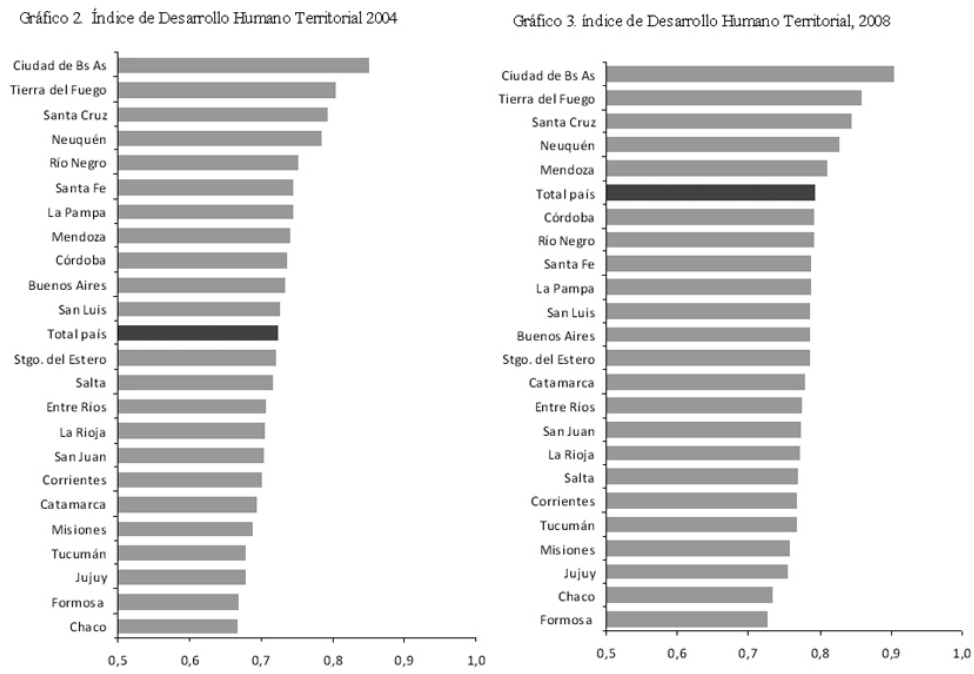
La evolución del IDHT entre 2004 y 2008 sugiere una transición hacia mayores niveles de desarrollo humano. Las cifras registradas se ubicaban en 2004 en un rango de variabilidad comprendido entre 0,850 para Ciudad de Buenos Aires- y 0,668 -para Chaco-. En 2008 se observa una modificación en la distancia entre posiciones extremas, siendo 0,905 el máximo -nuevamente para la capital nacional- y 0,726 el mínimo -esta vez para Formosa-. Así mismo, entre 2004 y 2008 la cantidad de provincias con IDHT alto (0,800 o más) pasó de dos (Capital Federal y Tierra del Fuego) a cinco (Capital Federal, Tierra del Fuego, Santa Cruz, Neuquén y Mendoza) el resto de circunscripciones se mantiene con un nivel de desarrollo humano medio.

Lo anterior muestra una amplia mejoría en el nivel de logros de las provincias; sin embargo, se hace necesario analizar el escenario de disparidades a partir de las modificaciones en las posiciones y la magnitud de los cambios que afectan a las jurisdicciones.

En cuanto a los movimientos registrados en las posiciones ocupadas en una y otra serie, se observa que Catamarca arroja la mejora relativa más significativa al pasar del puesto 18 al 13 entre 2004 y 2008, cambio que se explica principalmente, y como se verá más adelante, por el comportamiento del componente sanitario. Así mismo, Córdoba y Mendoza avanzan tres posiciones como consecuencia del incremento en sus logros durante el período analizado. Salta, por el contrario, pierde cuatro posiciones al trasladarse de la ubicación 13 a la 17, mientras que La Pampa, Río Negro y Santa Fe descienden dos lugares cada una. Las demás jurisdicciones mantienen su posición o bien experimentan pequeñas modificaciones que alteran el ranking de manera poco significativa.

Además del cambio en las posiciones relativas es interesante observar la magnitud de las variaciones entre uno y otro año. En este sentido, Tucumán arroja el cambio más significativo, habiendo aumentado su IDHT $13,4 \%$, por efecto, fundamentalmente, de la ampliación en los logros en salud y nivel de vida. Por la magnitud de sus variaciones también positivas le siguen Catamarca (12,2\%), Jujuy (11,4\%), San Juan (10\%) y Misiones (10\%). Es decir, las provincias que mayor incremento registran entre 2004 y 2008 pertenecen al grupo de jurisdicciones ubicadas en los últimos lugares del ranking. Esta situación representa como un proceso esperable, dado que las entidades más rezagadas poseen un margen más amplio de mejora de sus condiciones, es decir, esfuerzos relativamente reducidos generan cambios significativos en la situación de su población. Esto se corresponde con el comportamiento de las provincias más aventajadas en el IDHT, las jurisdicciones patagónicas y Ciudad de Buenos Aires, a las que se suma Santa Fe, presentan los menores porcentajes de variación en sus índices (entre 5\% y 7\%).

El examen del comportamiento de los componentes del IDHT arroja luz sobre los aspectos que estarían incidiendo en las modificaciones registradas en el nivel de logros de cada provincia. 


\section{Índice de Salud}

$\mathrm{Al}$ examinar el componente sanitario lo primero que se observa es una mejora generalizada en los índices provinciales, en mayor o menor medida la casi totalidad de entidades presenta un crecimiento positivo en el indicador, lo que implica un crecimiento del índice a nivel país de $12 \%$. Para el año 2004 los valores oscilan entre 0,928 y 0,526, cifras registradas por Tierra del Fuego y Formosa, la distancia entre ambas jurisdicciones es de 0,332 puntos. Los datos de 2008 arrojan un rango de variabilidad significativamente más estrecho, 0,242 puntos; siendo los valores extremos de 0,926 y 0,683 para las mismas entidades. Es decir, mientras en el primer año estudiado la situación de la provincia mejor posicionada superaba en 1,56 veces a la menos favorecida; al final del período esa distancia se había reducido a 1,36 veces, dando cuenta de un, aún insuficiente, proceso de convergencia hacia mayores niveles de logros.

Gráfico 4. Indice de SaLıd 2004

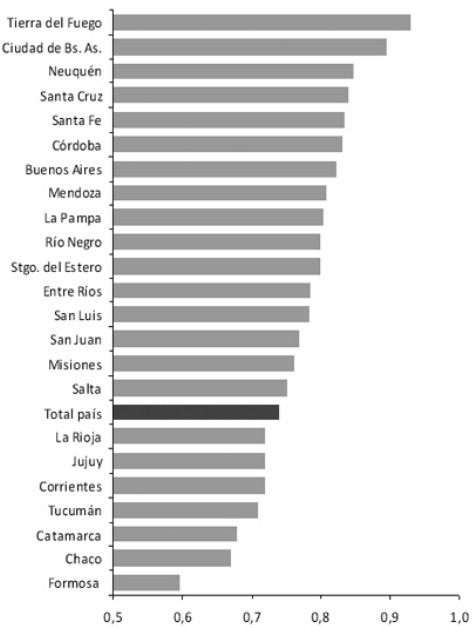

Gráfico 5. Indice de Sahud 2008

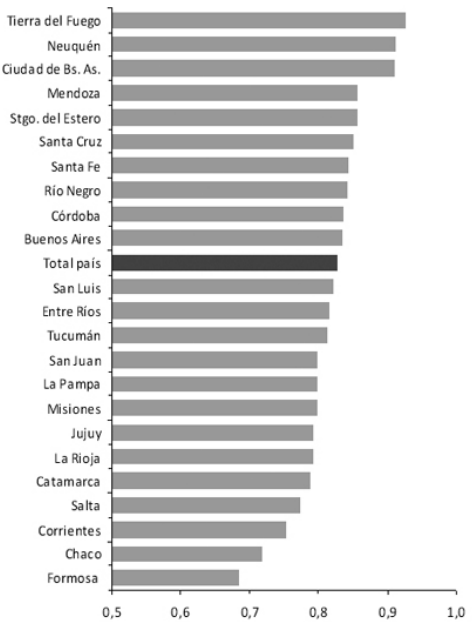

Tierra del Fuego ocupa, tanto en 2004 como en 2008, la primera posición en el ranking, la acompañan en la cima de la tabla Neuquén y Ciudad de Buenos Aires. Junto con esas jurisdicciones aparecen en el primer año de la serie Santa Cruz y algunas provincias del centro del país, Santa Fe, Córdoba, Buenos Aires y Mendoza. Ésta última mejora notablemente su posición relativa pasando a incorporarse en 2008 al grupo de las cinco provincias con mayores niveles de logros, mismo comportamiento tiene Santiago del Estero que en ese año se ubica detrás de Mendoza y por encima de Santa Cruz. 
El componente sanitario se presenta como un aspecto claramente deficitario en las provincias del norte del país, especialmente, en las pertenecientes a la región noreste. Formosa y Chaco, ocupan las posiciones menos ventajosas en ambas series de datos. Catamarca, Tucumán y Corrientes aparecían en 2004 formando parte de las cinco jurisdicciones con menores niveles de logros. En 2008, además de Formosa y Chaco, integran ese grupo Corrientes, Salta y Catamarca.

En el análisis comparado resulta notable la mejoría experimentada por Tucumán que asciende siete lugares en la clasificación; también registran ascensos importantes Santiago del Estero, que pasa de la ubicación 11 en 2004 a la 5 en 2008 y Mendoza que asciende cuatro lugares. Las variaciones en el ranking indican que La Pampa es la provincia que pierde mayor cantidad de lugares al retroceder del puesto 9 al 15 . Con ella, Salta, Córdoba y Buenos Aires registran las pérdidas relativas más significativas.

En cuanto a la magnitud de los cambios experimentados por las diferentes jurisdicciones en la dimensión salud, la provincia con mayor crecimiento en la tasa de sobrevivencia es Catamarca (16,2\%), seguida por Formosa (14,7\%), Tucumán (14,3\%), Jujuy (10,4\%) y La Rioja (10,0\%).

Esos datos completan el análisis respecto de los lugares en que se ubican las provincias en las clasificaciones relativas. En el caso de Formosa, aunque la misma ocupa la última posición en los dos ordenamientos, es una de las provincias que, en virtud de los esfuerzos realizados, ha logrado mejorar de manera más significativa las condiciones sanitarias de su población. Es justamente el rendimiento positivo de esa entidad el que incide principalmente en la reducción de la brecha de variabilidad dentro de la que oscilan los valores provinciales.

En contraste, La Pampa y Tierra del Fuego son las únicas jurisdicciones que experimentan una variación negativa en sus índices de salud ($0,5 \%$ y $-0,3 \%)$. Aunque hay que aclarar que estos poco significativos descensos pueden interpretarse más como un estancamiento de las tasas de sobrevivencia que como un deterioro de las mismas, especialmente en el caso de Tierra del Fuego que ha alcanzado valores prácticamente óptimos en el indicador.

Lo que merece la pena destacar respecto de las variaciones en el componente salud son los casos de Córdoba, Santa Fe y Buenos Aires, las provincias de mayor tamaño poblacional del país y las que registran las mejoras menos marcadas en los logros sanitarios entre 2004 y 2008. Córdoba sólo aumenta un $0,8 \%$ su índice de salud, mientras que Santa Fe y Buenos Aires lo hacen en magnitudes de 1,2\% y 1,5\% respectivamente. Salta y Misiones son también provincias que, aún con índices sanitarios relativamente bajos, registran un escaso crecimiento durante los años analizados, en el primer caso el incremento del indicador fue del 2,9\% y en el segundo de 4,7\%. Siendo el margen de mejora aún importante en todas 
estas jurisdicciones cabe esperar la realización de mayores esfuerzos para aumentar sus niveles de logro en la dimensión sanitaria.

\section{Índice de Educación}

Las clasificaciones generadas en el índice de educación muestran una amplia ventaja de Ciudad de Buenos Aires respecto del resto de jurisdicciones, la capital nacional se ubica en la primera posición con valores de 0,886 para 2004 y 0,939 para 2008 muy lejanos de las cifras alcanzadas por Salta, la segunda provincia en ambas clasificaciones (0,752 y 0,783 para uno y otro año). Si se excluye Ciudad de Buenos Aires y se considera la brecha existente entre Salta y Misiones (segundo y último puestos de las series), el resto de las provincias arroja una distribución significativamente homogénea, con pequeñas diferencias entre sus valores que oscilan en una distancia de 0,131 puntos en 2004 y 0,109 puntos en 2008.

El componente educativo brinda un panorama escasamente ajustado a la regionalización que señala una situación más favorable de las provincias patagónicas y la capital nacional respecto de las demás circunscripciones del país. En este caso, si bien Ciudad de Buenos Aires se ubica en una posición claramente ventajosa, las jurisdicciones de la región sur se distribuyen a lo largo de las clasificaciones sin mostrar correspondencia con el patrón de desarrollo regional antes señalado. En 2004 Neuquén, Tierra del Fuego y Santa Cruz arrojaban un relativamente buen rendimiento, mientras que Río Negro se ubicaba entre las cinco provincias peor posicionadas a la par de Entre Ríos, San Luis, Chaco y Misiones. En el año 2008 nuevamente Tierra del Fuego y Santa Cruz aparecen entre los primeros lugares de la clasificación y Río Negro vuelve a ubicarse a la cola del ranking, esta vez junto con Formosa, Chaco y Misiones.

Grifico 6 fndice de Educación 2004

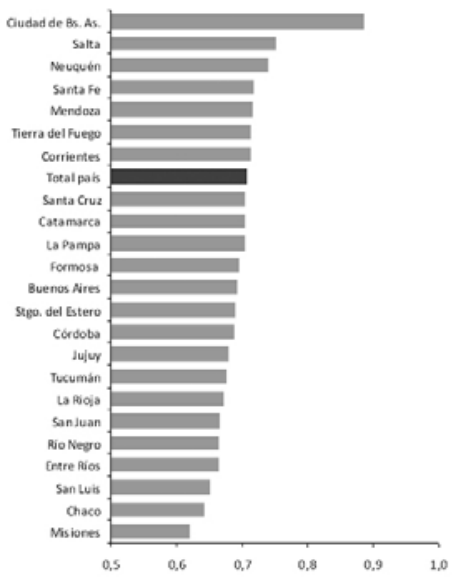

Crifico 7. findice de Educación 2008

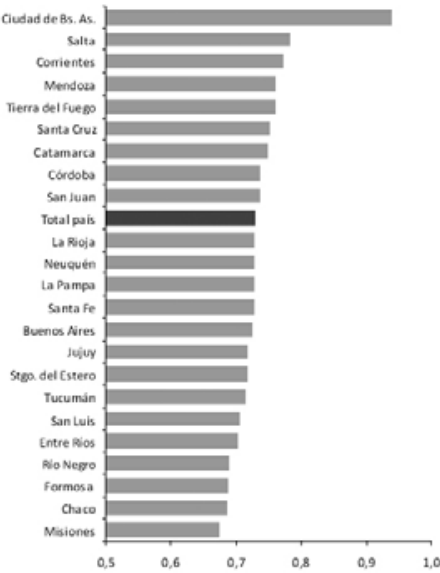


Destaca el rendimiento positivo de Salta y Mendoza, que se ubican en ambas series entre las cinco jurisdicciones con mayores niveles de logros. También notable es la posición favorable que ocupa Corrientes, que logra colocarse en el tercer puesto del ordenamiento 2008, esto sin olvidar que la información analizada refiere a la población residente en zonas urbanas.

Al estudiar las variaciones en las posiciones relativas entre 2004 y 2008 se observan en esta dimensión cambios notablemente más significativos que los registrados para el componente sanitario. Destacan el retroceso de diez lugares que experimenta Formosa, trasladándose de la posición 11 a la 21, la caída de Santa Fe desde la ubicación 4 a la 13 y la de Neuquén que pasa del lugar 3 al 11. En contraste, San Juan, La Rioja y Córdoba son las provincias que más puestos ascienden en la clasificación, nueve, siete, y seis posiciones respectivamente. Salta, Jujuy, Chaco y Misiones son las únicas jurisdicciones que mantienen su ubicación en ambas series, la primera permaneciendo en la segunda posición y las dos últimas a la cola de la clasificación.

En correspondencia con los cambios de posición registrados en la tabla, San Juan arroja el mayor crecimiento en el índice educativo, con un incremento de 10,8\%. Misiones (8,5\%), Corrientes (8,4\%), La Rioja (8,2\%) y San Luis (8,2\%) también experimentan importantes mejoras en la dimensión. Por su parte, Neuquén y Formosa ven reducidos sus valores en 1,4\% la primera y $1,1 \%$ la segunda, como en el componente sanitario, la escasa magnitud de esas cifras estaría indicando que el retroceso de estas jurisdicciones no respondería tanto a al deterioro de sus indicadores, sino fundamentalmente a su estancamiento y la pronunciada mejora relativa experimentada por otras entidades.

Entre las provincias que registran los menores niveles de crecimiento en sus logros educativos destacan Santa Fe (1,5\%), La Pampa (3,3\%), Santiago del Estero (3,8\%) y Río Negro (3,8\%).

Por la naturaleza de los indicadores utilizados en la construcción de este segundo componente, especialmente el de nivel educativo, y las ponderaciones otorgadas en el cálculo del índice, esta dimensión arroja, para prácticamente todas las provincias (excepto Ciudad de Buenos Aires), valores notablemente distantes del considerado óptimo. Los datos desagregados para las variables componentes indican que, en materia de logros educativos de la población en edad activa, todas las jurisdicciones tienen aún un largo camino por recorrer. Es de destacar en este sentido la situación de Misiones, Chaco y Formosa, algunas de ellas con importantes avances en los últimos años pero aún con notables deficiencias en cuanto a los logros educativos de su población urbana.

\section{Índice de Nivel de Vida}

En el índice de nivel de vida las provincias se ordenan de acuerdo con la regionalización que señala a las jurisdicciones patagónicas y Ciudad 
de Buenos Aires como las más aventajadas. En 2008 Santa Cruz mantiene el primer puesto que ocupa en 2004, nuevamente acompañada por Río Negro, Tierra del Fuego, Ciudad de Buenos Aires y Neuquén. En el extremo opuesto, con los menores índices, se ubican Santiago del Estero, Corrientes, Tucumán, Jujuy y Salta.

También en esta ocasión se evidencia una mejora generalizada en el nivel de logros. En 2004 el índice de Santa Cruz alcanza un valor de 0,833, distante en 0,198 puntos de Jujuy, la provincia peor posicionada. En 2008 las cifras extremas registradas en la dimensión son 0,929 y 0,752, para Santa Cruz y Salta, reduciéndose la brecha de variabilidad a 0,177. Por tanto, mientras que en 2004 la provincia mejor posicionada tiene un índice 1,31 veces mayor que la que ocupa el último lugar, en 2008 esa distancia se reduce a 1,24 veces.

Si se retoman las observaciones obtenidas para el componente educativo y se comparan con el comportamiento en esta tercera dimensión destacan, por sus situaciones contrapuestas, los casos de Río Negro y Salta. La primera, que registra un rendimiento positivo en el indicador de nivel de vida, se posiciona entre las jurisdicciones con menores niveles de logros en educación. A la inversa, Salta, que ocupa el último lugar en el tercer componente, se ubica en el segundo puesto según el valor obtenido en el índice educativo. Este desequilibrio estaría mostrando los desajustes que pueden existir entre las diversas dimensiones y la necesidad de generar estrategias que tiendan a articularlas y complementar los avances en las distintas áreas.

Gráfico 8 Índice de Nivel de Vida 2004

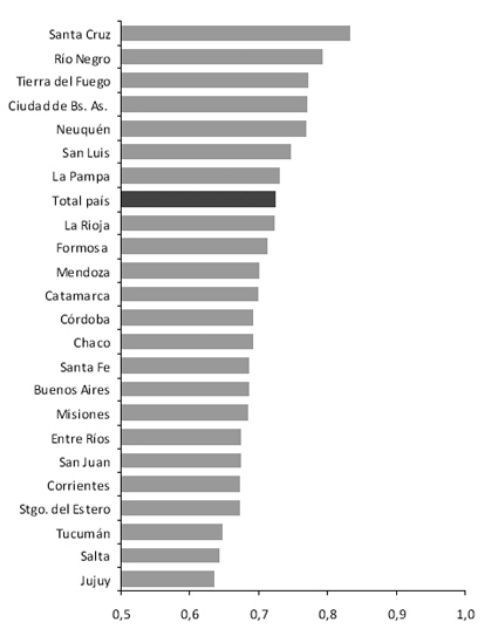

Gráfico 9 . Índice de Nivel de Vida 2008

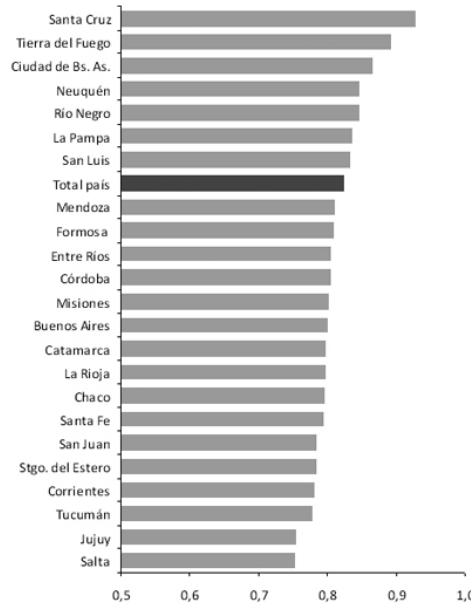


Las variaciones relativas que afectan a los índices provinciales de nivel de vida indican que La Rioja es la entidad que más lugares pierde, al retroceder del puesto 8 al 15 entre 2004 y 2008. Santa Fe, Río Negro, Chaco y Catamarca, caen tres lugares cada una. Entre Ríos es, en cambio, la jurisdicción que logra la mejora más significativa en su posición relativa, asciende siete lugares trasladándose de la ubicación 17 a la 10. Misiones, por su parte, avanza cuatro posiciones y Buenos Aires y Mendoza dos cada una.

Formosa, San Juan, Santa Cruz y Tucumán mantienen sus respectivos puestos en la clasificación. Entre esas entidades destaca el caso de Formosa, que ocupa el noveno lugar como consecuencia del alto valor de su indicador laboral, mientras que en las dimensiones sanitaria y educativa, como se vio anteriormente, se ubica entre las entidades con menor nivel de logros.

Para el componente de nivel de vida, los cambios más significativos se producen en las jurisdicciones que ocupan las posiciones centrales de ambos ordenamientos, no así en aquellas ubicadas en los extremos de las tablas. Tanto las cinco provincias con mayores índices en 2004 como las cinco con menores índices, permanecen formando parte de esos grupos en el año 2008.

Respecto de la magnitud de las variaciones de los índices, se obtiene que el indicador de nivel de vida registra los incrementos porcentuales más significativos en comparación con los otros dos componentes. A nivel país, el índice aumenta 13,8\%, pasando de 0,723 en 2004 a 0,823 en 2008. Tucumán es la provincia que mayor crecimiento experimenta en la dimensión $20,1 \%$ lo que, junto con su comportamiento positivo en el componente sanitario, explica la magnitud del incremento del IDHT de esa entidad. También importantes son los aumentos registrados por Entre Ríos y Jujuy que crecen 19,5\% y 18,7\% respectivamente. Otras once provincias se ven afectadas por notables incrementos en el componente (entre 15\% y 18\%).

Las provincias que menos aportan a la variación positiva del índice son Río Negro (6,8\%), Neuquén (10,0\%), La Rioja (10,3\%), San Luis $(11,4 \%)$ y Santa Cruz (11,5\%). A La Rioja este rendimiento insuficiente le supone el deterioro relativo ya mencionado, mientras que para las otras cuatro provincias implica cierto reacomodamiento en la clasificación pero sin perder sus posiciones relativamente ventajosas respecto del resto de jurisdicciones.

Estos análisis muestran que las posibilidades efectivas que tienen las personas de acceder a recursos materiales, aproximadas a través del ingreso per cápita e incorporando alguna aproximación a la distribución del mismo, dista de ajustarse a la riqueza acumulada en las diferentes jurisdicciones y evaluada a través del PIB per cápita. 


\section{Consideraciones finales}

El análisis precedente buscó dar cuenta del patrón de distribución territorial del desarrollo humano al interior de Argentina. Para ello, se partió de la aplicación de una versión ajustada del denominado IDHT, la nueva variante metodológica supone algunos cambios en los indicadores para aproximar cada dimensión y en los parámetros utilizados para su cálculo.

La aplicación del IDHT permitió obtener el mapa del desarrollo humano en Argentina y mostrar las significativas heterogeneidades existentes al interior de ese territorio nacional así como los avances y retrocesos ocurridos en el tiempo. En términos generales, la mayor exigencia de la nueva medida coloca al país entre las naciones de desarrollo humano medio, con un IDHT de 0,793 para 2008; cifra que resume la distancia que separa en 1,27 veces dos extremos, Ciudad de Buenos Aires y Formosa.

La diversidad capturada por los nuevos indicadores confirma que la región norte del país constituye un área prioritaria, siendo Chaco y Formosa las provincias más rezagadas. En el otro extremo, la capital nacional y las entidades patagónicas alcanzan los mayores niveles de logros. Esta regionalización se repite en los componentes sanitarios y de nivel de vida; pero aparece algo desdibujada en la dimensión educativa en la que, a excepción de Ciudad de Buenos Aires, el resto de jurisdicciones se distribuye sin responder a un patrón territorial.

El aspecto educativo se presenta como el más deficitario, por su valor agregado a nivel país, y el más desigualmente distribuido, por la amplia brecha que separa a Ciudad de Buenos Aires del resto de entidades, siendo Misiones la provincia que obtiene el índice más bajo en el componente.

Las observaciones más significativas se desprenden, sin embargo, de los cambios en el tiempo. Argentina ha experimentado en el período 2004-2008 una transición hacia mayores niveles de desarrollo. La novedad más importante es que las provincias que arrojan el mayor crecimiento provienen de la región norte, la más rezagada del país. El estrechamiento de las distancias interprovinciales indica que la transición estaría enmarcada entonces en un proceso de convergencia territorial.

Esas primeras apreciaciones merecen algunos matices, no todas las provincias marchan al mismo ritmo y las distancias entre ellas siguen siendo significativas. Entre 2004 y 2008 Tucumán, Catamarca y Jujuy arrojan el mayor crecimiento en sus IDHT y la dimensión nivel de vida registra los incrementos más significativos en comparación con los otros componentes.

En cuanto a las brechas interprovinciales, en 2008 el nivel de logros educativos de Ciudad de Buenos Aires es 1,39 veces el de Misiones, el índice de salud de Tierra del Fuego es 1,36 veces superior al de Chaco y el 
indicador de nivel de vida de Santa Cruz se distancia en 1,24 veces del de Jujuy. La equidad en la distribución de los logros sigue siendo aún un desafío por alcanzar.

Algunas cuestiones a resaltar respecto de las variaciones en cada dimensión son la escasa mejoría registrada en el componente sanitario por las provincias más pobladas -Córdoba, Santa Fe y Buenos Aires- y, en el lado opuesto, el significativo avance que en la misma materia ha logrado Formosa a pesar de ubicarse en la última posición del ranking. Santa Fe vuelve a destacar por su escaso crecimiento en el nivel de logros educativos, mientras que San Juan es la provincia con los mayores avances en ese componente. En el nivel de vida, Tucumán arroja el mayor incremento; mientras que las jurisdicciones que menos aportan a la variación positiva del índice son Río Negro, Neuquén, La Rioja, San Luis y Santa Cruz.

Los análisis realizados en este estudio brindan un aproximación empírica a la naturaleza territorial del desarrollo humano, demostrando que las capacidades se realizan y combinan de forma diferente en los distintos espacios y que, si bien existe cierta correspondencia en los logros alcanzados en cada dimensión, ninguna de ellas puede, de manera aislada, explicar el nivel de desarrollo de una determinada población. Esto exige seguir avanzando en herramientas conceptuales y metodológicas que contemplen la dimensión territorial y aporten conocimiento que sirva para el diseño y ejecución de políticas orientadas a la equiparación de oportunidades. 


\section{Notas}

${ }^{1}$ Pol (2008), Cividanes, Pol (2008).

${ }^{2}$ Pol (2008), Cividanes, Pol (2008).

${ }^{3}$ Para ampliar los fundamentos metodológicos que dan sustento a estos ajustes consultar Pol (2008).

${ }^{4}$ En aquellas unidades administrativas para las que la EPH cubre más de un aglomerado (caso de Buenos Aires) se promediaron los valores obteniendo como resultado la situación de la población urbana total de la provincia.

${ }^{5}$ En el año 2005 se incorpora al relevamiento el aglomerado chubutense Rawson - Trelew.

${ }^{6}$ OMS Estadísticas Sanitarias Mundiales 2009.

${ }^{7}$ OCDE Panorama de la Educación 2007 - Informe español.

${ }^{8}$ PNUD, Informe Mundial de Desarrollo Humano 2007-2008.

${ }^{9}$ PNUD, Informe Mundial de Desarrollo Humano 2007-2008.

${ }^{10}$ Rofman 1998, Vaca 2004, PNUD-Argentina 2005, entre otros. 


\section{Bibliografía}

Barreiro Cavestrany, Fernando (2007), Territorios virtuosos para el desarrollo humano. Competitividad, cohesión social y ciudadanía en el desarrollo local. II Encuentro Latinoamericano. Retos del Desarrollo Local. Gestión Innovadora de Territorios. Quito.

Cividanes, José Luis; Pol, María Albina (2008), Índice de Desarrollo Humano Territorial: Una propuesta metodológica. II Conferencia Latinoamericana y del Caribe sobre Desarrollo Humano y el Enfoque de las Capacidades Humanas, Montevideo.

INDEC (2004), Mercado de trabajo: Principales indicadores. Información de Prensa, Buenos Aires.

Ídem (2008), Mercado de trabajo: Principales indicadores. Información de Prensa, Buenos Aires.

Ministerio de Educación Ciencia y Técnica (2007), Panorama de la Educación. Indicadores de la OCDE 2007 - Informe Español, Madrid.

Ministerio de Salud (2005), Estadísticas Vitales. Información Básica - 2004. Buenos Aires.

Ídem (2009), Estadísticas Vitales. Información Básica - 2008. Buenos Aires.

OMS (2009), Estadísticas Sanitarias Mundiales 2009. Ginebra.

PNUD (2007), Informe Mundial sobre Desarrollo Humano 2007-2008. La lucha contra el cambio climático: Solidaridad frente a un mundo dividido, Nueva York.

PNUD-Argentina (2005), Informe de Desarrollo Humano 2005. Un tiempo de oportunidades. Argentina después de la crisis, Buenos Aires.

PNUD-México (2004), Informe de Desarrollo Humano 2004: El reto del desarrollo local, México D.F.

Ídem (2008),Índice de Desarrollo Humano Municipal en México 20002005, México D.F.

Pol, María Albina (2008), Desarrollo Humano y territorio: Una aproximación metodológica. Tesis Doctor en Economía, Repositorio Universidad de Alicante, Alicante.

Rofman, Alejandro; Romero, Luis (1998), Sistema socioeconómico y estructura regional en la Argentina. Amorrortu Editores, Buenos Aires. 
Rofman, Alejandro (2006), “El enfoque del desarrollo local: Conflictos y limitaciones”. En: Rofman, Adriana, Villar, Alejandro (comps.). Desarrollo local: Una revisión crítica del debate, Espacio Editorial, Buenos Aires.

Sen Amartya (2000), Un nuveau mòdele économique. Odile Jacob, Paris.

Vaca, Josefina (2004), “Articulación regional y desarrollo desigual en el territorio argentino”. Revista Territorios. 10-11:111-125, Universidad de Los Andes, Colombia.

Recibido: 05.03.2010

Aceptado: 03.12.2010 